\title{
Glottopol
}

Revue de sociolinguistique en ligne

Varia - Liberté, égalité, diversité !

\section{Éléments d'épistémologie pour l'étude des espaces ségrégués des banlieues urbaines sensibles de la région parisienne}

Marie-Madeleine Bertucci

\section{(2) OpenEdition}

Journals

Édition électronique

URL : https://journals.openedition.org/glottopol/565

DOI : $10.4000 /$ glottopol.565

ISSN : 1769-7425

Éditeur

Presses universitaires de Rouen et du Havre

Référence électronique

Marie-Madeleine Bertucci, «Éléments d'épistémologie pour l'étude des espaces ségrégués des banlieues urbaines sensibles de la région parisienne », Glottopol [En ligne], 33 | 2020, mis en ligne le 01 janvier 2020, consulté le 02 octobre 2021. URL : http://journals.openedition.org/glottopol/565 ; DOI : https://doi.org/10.4000/glottopol.565 


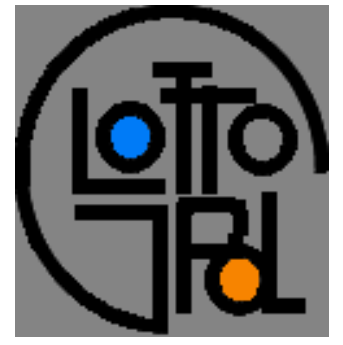

\section{GLOTTOPOL}

Revue de sociolinguistique en ligne $n^{\circ} 33$ - février 2020

Varia : Liberté, égalité, diversité !

\section{SOMMAIRE}

Hommages à Jean-Baptiste Marcellesi : À ringrazià vi, par Niculau Sorba ; “Ciao Ziu ! » par Philippe Blanchet.

Clara Mortamet : Présentation du numéro : Liberté, égalité, diversité !

Manon Him-Aquilli : "Prendre la parole sans prendre le pouvoir ». La mise en registre de l'horizontalité dans les assemblées générales anarchistes/autonomes.

Siham Hocini : La formulation du désaccord dans les débats télévisés algériens : quels "effetsde-politesse "?

Pauline Rannou : Trajectoires de la surdité en France. Parents entendants d'enfants sourds : questionner la fragmentation de l'identité sourde.

Catherine Combaz-Champlaine: Les rectifications orthographiques de 1990 comme révélateurs du rapport des enseignants à l'orthographe.

Marie-Madeleine Bertucci : Éléments d'épistémologie pour l'étude des espaces ségrégués des banlieues urbaines sensibles de la région parisienne.

Élise Gandon: Usages du numérique et illectronisme: nouvelles tâches pour le lecteurscripteur, nouveaux enjeux d'apprentissage.

Marc Arabyan : Quelle langue pour la recherche neurolinguistique française?

José R. de Arellano : Le rapprochement entre l'espagnol et le portugais pendant la décennie dorée sud-américaine : une problématique de l'horizontalité supercentrale.

Adam Wilson: Normes interactionnelles globalisées et communautés de pratique discontinues : les dynamiques sociolinguistiques du tourisme international.

Mélanie Lancien : Caractérisation de la variation liée à la situation de communication : apport de l'acoustique à la phonostylistique.

Assémou Maurice Ludovic Assémou : La prononciation du français parlé en Côte d'Ivoire. 


\title{
ÉLÉMENTS D’ÉPISTÉMOLOGIE POUR L'ÉTUDE DES ESPACES SÉGRÉGUÉS DES BANLIEUES URBAINES SENSIBLES DE LA RÉGION PARISIENNE
}

\author{
Marie-Madeleine Bertucci \\ Cy Cergy Paris Université, EA 7518 LT2D
}

Cet article se propose dans une perspective épistémologique de faire le point sur un certain nombre de notions essentielles à la compréhension d'une part des zones urbaines sensibles de la périphérie urbaine situées dans les banlieues défavorisées de la région parisienne ${ }^{1}$, qu'on définira comme des espaces urbains ségrégués et d'autre part à celle de leurs habitants. On s'intéressera aux représentations sociales attachées à ces derniers notamment dans le cadre de situations d'exclusion en postulant qu'il existe un faisceau de traits qu'on peut mettre en évidence et entre lesquels il est possible d'établir des relations. Ces traits permettraient de caractériser les zones urbaines sensibles et leurs habitants dans toute leur spécificité. Dans cette perspective, seront abordées dans un premier temps les caractéristiques des espaces urbains ségrégués de la banlieue, ensuite les représentations et les constructions sociales qui caractérisent leurs habitants et enfin la question de la culture de la pauvreté et de la désaffiliation envisagées sous l'angle de l'existence potentielle d'un risque de fragmentation du lien social dans ces espaces urbains ségrégués.

\section{Caractéristiques des espaces urbains ségrégués de banlieues ${ }^{2}-$ stigmatisation assignée de l'extérieur et statut ${ }^{3}$ minoritaire}

\section{I.1. Des espaces stigmatisés mais néanmoins minoritaires et altéritaires. Les banlieues urbaines sensibles}

Les banlieues urbaines sensibles de la région parisienne sont dotées d'une image péjorative, qui fonctionne comme un contrepoint négatif de l'espace urbain des quartiers résidentiels

\footnotetext{
${ }^{1}$ On se limitera ici à l'hexagone.

${ }^{2}$ Voir sur ce point Bertucci $(2009,2012,2020)$.

${ }^{3}$ Le statut est défini en sociologie de la manière suivante : «Pour la sociologie classique américaine, le statut (ou status) désigne la position qu'un individu occupe dans un système social donné. Associé à un ensemble de rôles, il définit ce que l'individu est en droit d'attendre du comportement des autres à son égard. Si toutes les positions ne sont pas nécessairement liées à une hiérarchie, les sociétés se caractérisent généralement par des classements statutaires dont les fondements dépendent de leurs systèmes de valeurs. Les statuts sont donc ordonnés selon le niveau de privilèges et d'honneur qu'ils confèrent aux individus. S'intéressant aux conséquences sociales de cette distribution, Weber estime que les individus occupant une même position dans le système de prestige d'une société
} 
dépourvus eux des mêmes désavantages économiques et sociaux. La représentation dominante de ces espaces, souvent stéréotypée et parfois démentie par la réalité des lieux est celle de lieux dénués de valeur esthétique, bruyants, où se concentrent les nuisances urbaines (Rey, 1996 : 17). Cette représentation se déploie à partir d'une approche par l'espace qui amène à poser en ces termes les problèmes sociaux, notamment ceux de l'intégration des migrants et les inégalités sociales dont ils sont frappés. Cette démarche spatialiste - production par l'espace de problèmes sociaux - et localiste - confusion de l'espace et des attitudes et identités de ceux qui s'y trouvent - (Baudin, Genestier, 2002 : 11) renvoie au fait que parler de la banlieue conduit à évoquer inéluctablement un problème social tout en l'euphémisant pour éviter d'évoquer des questions problématiques. Cet ensemble de traits est révélateur du statut minorisé de ces

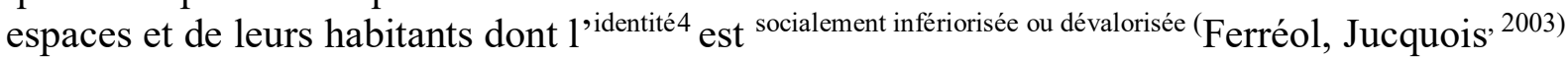
du fait de la pluralité de leurs ${ }^{\text {désavantage }}{ }_{S}$ qu'ils soient ${ }^{\text {démographique }}{ }$, économiques, ${ }^{\text {politique }}$, sociaux et ${ }^{\text {culturel }}$ s. On en veut pour preuve le fait que de nombreux indicateurs attestent des manifestations de l'exclusion dans les espaces urbains ségrégués des banlieues et en particulier les indicateurs socioéconomiques, lesquels sont corrélés avec la présence visible de migrants parmi les habitants de ces espaces. On citera pour illustrer ces différents points cet extrait des premières lignes de l'Avant-propos de la synthèse du rapport 2011 de l'ONZUS ${ }^{5}$ :

Plus d'une personne sur deux résidant en $Z_{U S}^{6}$ est immigrée ou descendante d'immigrés. La situation socioéconomique de ces personnes est sensiblement plus difficile que celle du reste de la population notamment en ZUS: elles sont plus exposées au chômage, occupent plus souvent des emplois moins qualifiés et touchent des salaires moins élevés. Elles sont également moins mobiles.

\footnotetext{
forment des groupes statutaires (status groups) : ils ont en commun sentiment d'appartenance, style de vie et point de vue sur le monde. Les groupes statutaires renvoient ainsi à la dimension culturelle de la stratification sociale aux côtés des classes économiques et des partis politiques. Si la construction théorique du statut social est au cœur de conceptions diverses et en débat, on considère généralement que la position sociale des individus participe de l'explication des comportements, attitudes et aspirations. Le plus souvent, le statut social constitue un indicateur synthétique de la position socio-économique des individus, dont la profession est un élément central. Les sociologues cherchent à le mesurer et l'utilisent comme variable dans leurs analyses. Il est aussi au cœur d'analyses plus dynamiques en termes de mobilité sociale et de déclassement. De façon plus restrictive, dans la nomenclature des PCS [= nomenclature des professions et catégories socioprofessionnelles] de l'INSEE, le statut renvoie à l'un des critères de classement et permet de distinguer les salariés et les non-salariés (patrons, artisans, agriculteurs, commerçants) » (Rui, in Paugam, $2015: 115$ ).

${ }^{4}$ On définira l'identité comme «l'ensemble des caractéristiques et des attributs qui font qu'un individu ou un groupe se perçoivent comme une entité spécifique et qu'ils sont perçus comme telle par les autres. Ce concept doit être appréhendé à l'articulation de plusieurs instances sociales, qu'elles soient individuelles ou collectives. L'identité personnelle est le produit de la socialisation, laquelle permet la constitution du «Soi ». Pour les sociologues interactionnistes, les identités individuelles naissent des interactions sociales plus qu'elles ne les précèdent. L'identité n'est pas une propriété figée, c'est le fruit d'un processus. Ainsi, le travail identitaire s'effectue de manière continue tout au long de la trajectoire individuelle et dépend à la fois du contexte et des ressources qui peuvent être mobilisées. Cette identité se modifie donc en fonction des différentes expériences rencontrées par les individus. Claude Dubar distingue deux composantes indissociables de l'identité sociale. L'« identité pour soi » renvoie à l'image que l'on se construit de soi-même. L'«identité pour autrui » est une construction de l'image que l'on veut renvoyer aux autres; elle s'élabore toujours par rapport à autrui, dans l'interaction, en relation avec l'image que les autres nous renvoient, c'est une reconnaissance des autres. Les identités collectives trouvent leur origine dans les formes identitaires communautaires où les sentiments d'appartenance sont particulièrement forts (culture, nation, ethnies...) et les formes identitaires sociétaires qui renvoient à des collectifs plus éphémères, à des liens sociaux provisoires (famille, groupe de pairs, travail, religion...). L'individu appartient ainsi de manière simultanée ou successive, à des groupes sociaux qui lui fournissent des ressources d'identification multiples » (Castra, in Paugam, 2015 : 72-73).

${ }^{5}$ Observatoire national des zones urbaines sensibles.

${ }^{6}$ Zone urbaine sensible.
} 
Une note de France Stratégie ${ }^{7}$ confirme cette analyse quatre ans plus tard :

Ces difficultés sont identifiables en matière d'éducation, d'emploi, de conditions de vie et de logement; elles sont particulièrement marquées pour certaines catégories de population $^{8}$, dont les enfants ayant deux parents immigrés, les descendants d'immigrés d'Afrique, les garçons. Ces difficultés reflètent d'abord la situation socioéconomique de ces jeunes et de leurs parents, exposés aux défaillances de nos politiques publiques: obstacles à l'entrée sur le marché du travail des jeunes et des peu qualifiés, réussite scolaire tributaire de l'origine sociale, absence de fluidité du marché du logement, existence de discriminations. Les constats sont similaires pour les habitants des quartiers de la politique de la ville. (Cusset et al., $2015: 1$ )

Les inégalités socioéconomiques, qui pèsent sur les groupes de migrants, ont pour conséquence leur relégation dans les espaces défavorisés de la banlieue urbaine sensible, du fait de leurs faibles possibilités d'accès à des espaces mieux dotés et de leur présence visible dans les zones les plus vulnérables. De ce fait, les désavantages qu'on vient d'évoquer sont transférés par les groupes concernés aux quartiers où ils résident et au type d'habitat qui prévaut dans ces quartiers. Quartier et habitat en viennent à constituer des manifestations supplémentaires d'exclusion et certains des traits emblématiques des zones urbaines sensibles. On notera que le terme «quartiers » au pluriel apparait dans le discours commun pour désigner les cités défavorisées de la banlieue.

\section{I.2. Le quartier, l'habitat : des manifestations de l'exclusion}

\section{Le quartier}

La notion de quartier ${ }^{9}$ doit être prise en compte si on admet que le quartier constitue un espace intermédiaire entre le logement et la ville et permet d'aborder la question du système résidentiel urbain et de son évolution (Hoggart, 1957 ; Baudin, Genestier, op. cit. : 46), les banlieues urbaines sensibles auxquelles on s'intéresse dans cet article étant au bas de la hiérarchie des lieux résidentiels. La dénomination est aussi utilisée par les habitants eux-mêmes avec une intention non stigmatisante pour les cités mentionnées (Bertucci, 2009 et 2017). Elle est analysée par David Lepoutre comme une marque d'attachement résidentiel (1997: 51). Le quartier articule social et spatial, il construit de ce fait un espace public fondé sur l'interconnaissance, la mixité, la diversité fonctionnelle et constitue par là même un espace d'intégration, de socialisation ${ }^{10}$ (Bertucci, 1995: 158; Bertucci, 2006 : 122), un cadre de

\footnotetext{
${ }^{7}$ Cette publication hors série de mars 2015 émane du Commissariat général à la stratégie et à la prospective.

${ }^{8}$ Voir aussi sur ce point Lagrange (2010).

${ }^{9}$ La notion de quartier est contestée par un courant de la recherche en sciences sociales car elle parait floue et qu'elle s'applique à des espaces très différents les uns des autres : quartier bourgeois, anciens quartiers ouvriers des banlieues populaires...

10 On proposera la définition suivante de la socialisation: "La socialisation désigne les mécanismes de transmission de la culture ainsi que la manière dont les individus reçoivent cette transmission et intériorisent les valeurs, les normes et les rôles qui régissent le fonctionnement de la vie sociale. La socialisation «manifeste» peut être assimilée à un processus volontaire et explicite visant à structurer la personnalité d'autrui. La socialisation « latente » correspond davantage à un processus où l'enfant intériorise les normes et les valeurs de la société dans laquelle il vit sans qu'il y ait d'apprentissage spécifique ni réelle conscience de participer à ce processus. [...] La socialisation doit être considérée comme un processus continu qui concerne les individus tout au long de leur vie. On distingue classiquement une socialisation primaire et une socialisation secondaire. La socialisation primaire correspond à la période de l'enfance. Ce processus s'effectue d'abord dans la famille qui en constitue l'instance principale; son action est essentielle pour la structuration de l'identité sociale. L'école représente une autre instance majeure de la socialisation primaire : pour Émile Durkheim, cette socialisation méthodique de la jeune génération par la génération adulte permet d'inculquer les normes et les valeurs qui constituent le fond commun de la société. L'enfant se socialise également de manière plus informelle à travers le groupe des pairs. La socialisation secondaire se fonde sur les acquis de la socialisation primaire, les prolonge et éventuellement les
} 
développement de solidarités ${ }^{11}$ locales et de sociabilités de proximité (Baudin, Genestier : 44). David Lepoutre souligne à ce sujet la « circonscription symbolique » (1997: 51 et suiv.) qui en résulte, qu'il attribue à l'existence de solidarités primaires qui se tissent entre certains habitants. Ces solidarités sont fondées sur des regroupements communautaires, qui se sont construits avec l'arrivée des familles de migrants et leur installation dans les logements des cités de logements sociaux des quartiers défavorisés des banlieues ségréguées.

L'habitat est un second facteur, après le quartier, de stigmatisation.

\section{L'habitat}

Même si l'habitat en banlieue est varié et se développe aussi bien à travers des zones pavillonnaires que des grands ensembles ou des cités, il possède des traits caractéristiques souvent stéréotypés pour l'opinion commune. En effet, c'est l'image connotée négativement des cités HLM qui s'impose au détriment de celles dont sont dotées les banlieues bénéficiant d'un niveau de vie élevé et ne connaissant pas de problèmes sociaux de la même ampleur. Les cités de banlieues ou les grands ensembles ont fait l'objet de représentations symboliques fortes en termes d'unité et de clôture, qui résultent de leur architecture spécifique, récente et non monumentale, emblématisée par les «barres » des cités HLM (Lepoutre, 1997 : 54 et suiv.) dont Lepoutre considère qu'elles constituent une « clôture de l'espace » qui constitue le pendant concret de la « circonscription symbolique » évoquée plus haut à propos du quartier (1997: 51 et suiv.).

L'examen de ces deux notions conduit à dire que la question de l'exclusion apparait comme un thème central de la notion d'espaces urbains ségrégués et des thématiques afférentes, à savoir la vulnérabilité, la précarité, la relégation... surtout si on admet que l'exclusion constitue un marqueur de décohésion sociale (Guibet Lafaye, 2012 : 29) qui peut s'exercer à deux niveaux. À l'échelle d'un quartier, d'une cité, voire d'une commune, l'exclusion parait être un mécanisme social, un mode de fonctionnement de la société (Ibid.) spécifiquement repérables, qui contribuent au maintien d'individus, voire de groupes dans une situation de relégation (Ibid.) en affaiblissant les solidarités objectives (op. cit. : 30). Appliqué à un individu, le degré d'exclusion se mesure en fonction d'une échelle ${ }^{12}$ qui hiérarchise ses possibilités d'accès aux sphères décisionnelles, économiques, politiques, culturelles en conformité avec des mécanismes sociaux identifiables (op. cit. : 28). Une moindre participation subie, qui ne serait

transforme. Elle permet aux adultes de s'intégrer à des groupes spécifiques (travail, association, parti politique...) ; chaque individu est ainsi socialisé aux différents rôles sociaux et aux statuts qui seront les siens au cours de sa vie. Si elle est particulièrement intense pendant l'enfance, la socialisation n'est donc jamais achevée, ses résultats sont provisoires et toujours susceptibles d'être remis en question. » (Castra, in Paugam, 2015 : 97-98)

${ }^{11}$ On renvoie à la définition qui suite de la notion de solidarité : «Le concept de solidarité est l'un des premiers mots de la sociologie : Émile Durkheim s'appuie sur la distinction entre la «solidarité mécanique » et la « solidarité organique » pour analyser l'évolution des sociétés modernes. La solidarité mécanique fonde le lien social au sein des sociétés traditionnelles; elle dérive principalement de la similitude des membres du groupe et de leurs fonctions. Durkheim considère qu'à mesure que les fonctions sociales se spécialisent et se diversifient, une solidarité organique se substitue à cette solidarité mécanique. Elle se fonde au contraire sur une différenciation des tâches qui inscrit les individus dans des liens d'interdépendance sociale. Autrement dit [dans] une société moderne, les membres du groupe sont certes spécialisés, mais complémentaires. Dans les travaux sociologiques plus récents, la notion de solidarité reste intimement imbriquée à celle de lien. Elle en épouse donc les différentes formes : familiales, intergénérationnelles, sociales, citoyennes... Chargé des termes du débat public, ce concept polysémique appelle à être déconstruit du point de vue sociologique, au profit non seulement d'une lecture des pratiques de solidarité et de leurs manifestations multiples, mais aussi de leurs fondements, c'est-à-dire des formes d'échange et des logiques de réciprocité dans lesquelles elles s'inscrivent. Ainsi déconstruit, le terme de solidarité devient un outil conceptuel majeur dans la comparaison des modèles sociaux : il permet l'analyse de la variabilité des formes de solidarité à différents échelons territoriaux et sociaux, et de la façon dont elles s'articulent au sein des sociétés contemporaines. » (Van de Velde, in Paugam, 2015 : 99)

${ }^{12}$ Selon Guibet Lafaye, l'analyse en termes de degrés ou de participation est un cadre théorique pertinent pour étudier la notion d'exclusion (Guibet Lafaye, $2012: 28$ ). 
pas la conséquence d'une décision individuelle, est interprétable comme une situation d'injustice sociale, comme la manifestation de l'absence de parité dans l'accès aux sphères décisionnelles et le résultat d'obstacles arbitraires (Ibid.). Cette stigmatisation et cette minorisation rendent difficile l'autonomisation sociale et renvoient ces groupes à une altérité génératrice d'exclusion. Les faits d'exclusion attachés aux individus et à leurs modes de relations sociales feront l'objet de la seconde partie de cet article consacrée à la question des habitants des zones urbaines sensibles.

\section{Les habitants}

\section{Représentations et constructions sociales}

On observe des faits de ségrégation et d'exclusion dans ces espaces urbains ségrégués, qui peuvent être interprétés comme des supports de domination. On fera l'hypothèse qu'ils résultent de représentations liées à l'origine et en particulier à des représentations de l'ethnicité, qu'on analysera comme un ensemble de «constructions sociales » ${ }^{13}$. Ces représentations de l'ethnicité résultent de l'installation de familles migrantes dans ces espaces, qui a eu pour conséquence le processus d'ethnicisation des classes populaires (Boucher, 2012: 25), qui constitue un facteur de stigmatisation supplémentaire des zones urbaines sensibles concernées.

Cette situation peut expliquer des formes de repli sur soi, qui vont être évoquées dans les lignes qui suivent et qui contribuent à renforcer les solidarités construites à partir de l'appartenance à des communautés qui se construisent peu ou prou en se référant à une dimension ethnique partagée.

\section{II.1 L'ethnicité}

La notion d'ethnicité constitue le produit d'un certain nombre de « constructions sociales ${ }^{14}$ par la mise en exergue de caractéristiques chargées d'emblématiser de soi-disant différences entre des groupes d'individus et de les réifier comme minorités naturelles » (Cognet, 2010 : 103) du fait de l'essentialisation et de la réification de leurs caractéristiques. Marguerite Cognet, à ce propos, souligne que le discours commun comprend la notion d' " assignation ethnique » comme « une différence de pratiques culturelles » et celle d' « assignation raciale ${ }^{15} »$ comme $^{2}$ « une différence inscrite dans le biologique, reliée plus ou moins explicitement à des attributs phénotypiques » (Cognet, $2010: 103)$. Elle précise ${ }^{16}$ que :

Ces constructions sociales de différences procèdent de la racisation des individus qui n'est autre que l'instruction d'une altérité radicale par essentialisation de toutes sortes de traits culturels ou biologiques attribués, nolens volens, à l'ordre de la nature des choses (Ibid.).

Cette catégorie qu'est l'ethnicité fonctionne comme une "prophétie autoréalisatrice ${ }^{17}$ » (Cognet, 2010 : 103) et s'impose comme une « injonction de conformité » et un « rôle » (Ibid.)

\footnotetext{
${ }^{13}$ Ces constructions sociales ont pu être héritées. Certains groupes bien identifiés ont été mis en altérité et ont permis à certains Européens de se construire une identité positive par le biais de différenciations discriminantes (Joffe, 2005 : 96) lesquelles ont été développées par certains auteurs. On peut penser ici notamment à la théorie des races de Gobineau, qu'il traite dans son Essai sur l'inégalité des races humaines (1884). Ce texte a contribué à la formation des théories du nazisme (Young, 1995).

14 À l'instar d'autres catégories comme le genre ou la classe. On peut se reporter également à Elsa Dorlin (2008).

${ }^{15}$ Isabelle Clair $(2015$ : 113) souligne que «le terme « race» souvent perçu comme une importation nordaméricaine, fait débat en France. Ses détracteur(trice)s, qui ne nient pas l'existence de groupes sociaux racisés, lui préfèrent souvent « ethnicité » : terme moins connoté et dépourvu de référence au biologique ».

${ }^{16}$ Voir aussi sur ce point Colette Guillaumin (2002).

${ }^{17}$ Voir sur ce point Robert King Merton (1997).
} 
aux individus assujettis à ce type de regard social. Françoise Lorcerie note à ce sujet que le recours au critère ethnique pour désigner l'autre sous-entend " une auto-définition ethnique de soi - assumée ou non (en France, c'est l'idée de « Français de souche ») » (2009: 65).

Elle peut être également à l'origine de processus d'identification à l'espace. On proposera de désigner ce processus comme une identité territorialisée qui prendrait la forme d'une ethnicité territoriale (Ibid.) susceptible de constituer un facteur de stigmatisation supplémentaire. On a pu parler à cet égard de « quartiers d'exil » (Dubet, Lapeyronnie, 1992) du fait de la « concentration » de populations non autochtones dans certaines villes ou quartiers défavorisés sur le plan économique et social (Lorcerie, 2009: 64). Cette concentration est génératrice de ségrégation, c'est-à-dire d'un «creusement de distances entre populations autochtones et populations vues comme d'origine différente » (Ibid.). F. Lorcerie analyse le lien entre l'espace stigmatisé et la présence au sein de cet espace de populations non autochtones comme une forme de ce qu'elle nomme l'ethnicité territoriale (2009:65) comme l'illustrent les propos suivants :

Pour résumer, l'" ethnicité territoriale " renvoie à la formation d'une configuration sociale inscrite dans un espace où des facteurs sociaux (concentration de populations démunies, manque d'emploi, dépréciation du lieu pour diverses raisons) sont combinés aux facteurs intersubjectifs de la visibilité ethnique (réactions identitaires diverses). (Lorcerie, $2009: 65)$

La relégation n'est pas un phénomène nouveau mais les émeutes de 2005 ont révélé les difficultés de certains citoyens français, qui se perçoivent et qui sont amenés à se percevoir comme illégitimes du fait d'une société à laquelle pourtant ils appartiennent, dont ils sont citoyens, mais qui continue à les voir comme différents.

\section{2 Ethnicité et relégation}

Didier Fassin dans un entretien accordé à la revue L'Histoire ${ }^{18}$ montre que la loi française ne reconnait pas la notion de race, n'impose pas de barrière à la circulation et n'organise pas le découpage ethnique de la population comme cela a pu être le cas en Afrique du Sud par exemple. Cela n'empêche pas néanmoins qu'existent en banlieues des concentrations communautaires de populations pauvres dans les cités (Ibid.). À quels critères faut-il attribuer ces processus de discrimination, cette mise en altérité, qui essentialisent la différence? Nationalité, couleur de peau, patronyme, variété de français, pratiques linguistiques ? Fassin pose la question de savoir si le critère implicite mais puissamment actif n'est pas une forme de discrimination liée à l'origine qui ne se dit pas. Ces discriminations n'impliquent pas nécessairement de racisme mais lorsqu'elles se manifestent, elles font porter aux personnes concernées la responsabilité de la discrimination, ce qui évite d'aborder la question en termes politiques et économiques. Les difficultés évoquées sur le plan de l'emploi sont développées dans des publications au sujet notamment des jeunes diplômés issus de l'immigration et de leurs difficultés à trouver un emploi (Tavan, 2005; Frickey, 2005; Mucchielli, Le Goaziou, 2006 : 28). La question de la discrimination au faciès est d'autant plus préoccupante que les revendications des acteurs de banlieues relèvent davantage de la sphère de la citoyenneté que de la sphère de l'ethnicité (nationale, linguistique ou confessionnelle) (Wacquant, $2006: 292$ ). Il s'agit plus de la revendication d'une égalité en matière d'emploi, de logement, d'éducation, de santé, dans les rapports avec la police. On peut y voir le signe que les revendications des acteurs ne traduisent pas des manifestations d'identités communautaires ethniques organisées et demandant à être reconnues comme telles dans l'espace public. Pour conclure sur ce point, on dira que le problème n'est pas apparu avec la crise de 2005. Didier Lapeyronnie soulignait

\footnotetext{
${ }^{18}$ «Y a-t-il vraiment des discriminations raciales en France ?». L'Histoire, 306, février 2006.
} 
déjà en $2004^{19}$ que l'absence d'organisation communautaire n'empêche pas qu'individuellement, les acteurs des banlieues se construisent parfois selon des appartenances communautaires. Néanmoins, force est de constater que le facteur de l'ethnicité conduit ou peut conduire à des modes d'organisation communautaires et à un repli sur eux-mêmes des groupes concernés qui développent de ce fait une forme spécifique de lien social ${ }^{20}$.

${ }^{19}$ Le Monde du 6 juillet 2004.
${ }^{20}$ On définira ainsi la notion de lien social : « La notion de lien social est faussement simple à saisir. Très souvent mobilisée sans être définie, elle renvoie cependant à des mécanismes complexes dont l'analyse est au cœur de la pensée sociologique. Dès ses débuts la sociologie a montré l'évolution des liens sociaux dans un contexte d'individualisation croissant. L'analyse du lien social et de son évolution à la fin du XIX ${ }^{\mathrm{e}}$ siècle est inséparable de la compréhension du changement social, qui touche la société de l'époque. Serge Paugam propose de réfléchir à partir de la thèse d'Emile Durkheim, De la division du travail social, soutenue en 1893. Ce dernier analyse le changement social en portant son regard au niveau des liens de solidarité. Les sociétés traditionnelles, à dominante agraire, étaient caractérisées par la solidarité mécanique organisée par la communauté locale entre des individus qui partagent les mêmes valeurs et se conforment aux règles collectives. La conscience collective que Durkheim définit comme «l'ensemble des croyances et des sentiments communs à la moyenne des membres d'une même société » imprègne davantage les comportements dans les sociétés traditionnelles. La modernité se traduit par une solidarité organique, fondée sur la complémentarité et l'interdépendance des individus et des fonctions sociales qu'ils remplissent. Cette complémentarité, qui résulte de la division du travail social est rendue possible par un affaiblissement de la conscience collective. Le sociologue allemand Ferdinand Tönnies avait également posé la question de la transformation du lien social dès 1887. Il oppose deux types idéaux, la communauté propre aux sociétés traditionnelles, au sein desquelles la proximité et l'interconnaissance de ses membres est forte ; la société est quant à elle impersonnelle, les relations en son sein sont contractuelles et non plus fondées sur la seule confiance. Max Weber propose d'analyser la transformation du lien social en distinguant les rationalités traditionnelles et «affectuelles » des sociétés traditionnelles, des rationalités en valeur et en finalité des sociétés modernes. [...] Dans les sociétés modernes : «l'autonomie croissante de l'individu débouche sur des interdépendances plus étroites avec les autres membres de la société » (Paugam, 1988). La solidarité organique se met en place de façon institutionnelle, assurant à tous une plus grande protection contre les risques sociaux. Cependant, ce mouvement est progressif et va toucher l'ensemble des liens qui rattachent l'individu à la société. La communauté perd son rôle protecteur, l'individu peut alors s'en émanciper, investissant différemment les institutions traditionnelles. Il faudra attendre l'année 1946 instituant le système de protection sociale généralisée pour voir la solidarité collective appliquée à tous. Avant la guerre, l'idée d'une protection sociale assurée par des organismes d'assurances se développe en lien avec l'essor du salariat. Cette histoire spécifique de la France explique la dualité de son système de protection sociale, à la fois assuranciel, adossé au travail et universel financé par l'État. [...] [On observe ensuite une évolution qui se traduit par le] passage « Du lien social aux liens sociaux » (Paugam, 1988). En effet, alors que dans les sociétés à solidarité mécanique les individus étaient inscrits dans un cercle étroit de socialisation, les sociétés modernes se caractérisent par la pluralité des liens reliant les individus au monde social. Les travaux de Georg Simmel et Norbert Elias ont mis en évidence la pluralité des appartenances, analysant le lien social des sociétés modernes comme l'entrecroisement de plusieurs liens. [...] La construction d'une identité est un enjeu essentiel dans les sociétés modernes. Elle repose sur l'inscription dans un ensemble de liens sociaux qui assurent à l'individu la reconnaissance d'autrui. L'auteur propose une typologie de quatre types de liens sociaux principaux (le lien de filiation, celui de participation élective, le lien de participation organique entre acteurs de la vie professionnelle, et le lien de citoyenneté) indiquant la forme de protection de reconnaissance associée. [...] Les sociologues contemporains [ont mis en évidence] les risques et les conséquences individuelles de la rupture des liens sociaux. Les conséquences de la rupture d'un lien sont variables d'un individu à l'autre. Les plus défavorisés n'ont souvent que peu de ressources pour tisser et entretenir des liens suffisamment nombreux capables de leur assurer une stabilité. [La période contemporaine voit] le retour de l'insécurité sociale. La question de la cohésion sociale est au centre de cette réflexion. En effet, le principe de la solidarité organique est mis à mal depuis le durcissement des conditions de vie dans les années 1980 lié à l'explosion du chômage et de la précarité salariale. L'auteur indique qu'entre 1993 et 2001 l'explication de la pauvreté par la paresse dans l'ensemble des pays européens a fortement cru alors que l'explication par l'injustice sociale a fortement décliné. Cette stigmatisation masque la question de la place des plus désavantagés dans le système social. Aujourd'hui les moins qualifiés sont aussi les moins bien protégés, les moins stables professionnellement, et ceux qui bénéficient le moins de la formation continue. L'école joue aussi un rôle important dans la reproduction des inégalités sociales et économiques. « Est-ce normal que les enfants des milieux défavorisés soient condamnés à échouer dans le système scolaire sans avoir la chance d'acquérir les bases nécessaires à leur intégration sociale ?» (Paugam, 1988). Pour répondre à ces problèmes, [Serge Paugam] préconise de ne pas opposer les politiques universalistes et catégorielles. Il propose ainsi de corriger temporairement les principes universalistes par des mesures catégorielles. 


\title{
II.3 Les modes d'organisation communautaire des populations migrantes. Une forme spécifique de lien social caractérisée par des formes de repli communautaire.
}

\author{
Le repli communautaire est en général expliqué par le recours à la notion d'endogamie.
}

\section{II.3.1 La notion d'endogamie suffit-elle pour l'analyse des faits de repli communautaire?}

On peut expliquer l'endogamie par l'absence de cohérence démographique, d'unité culturelle et d'organisations représentatives fortes, ce que Wacquant décrit comme « l'absence de parallélisme institutionnel » (Ibid.). Il en résulte une déstructuration du lien social qui autoriserait des modalités d'organisation communautaire, caractérisées par une endogamie et un repli sur des traditions culturelles, d'après certains observateurs ${ }^{21}$. Un rapport des Renseignements Généraux de 2004, cité par le quotidien Le Monde, notait déjà la tendance à l'endogamie dans certains groupes avec le maintien de survivances culturelles traditionnelles et une forme de régulation sociale parallèle, susceptible de conduire à un repli communautaire. Cette notion d'endogamie a néanmoins été mise en débat. La tendance au repli communautaire n'est pas discutée mais elle est attribuée à d'autres facteurs et en particulier à une situation socio-économique spécifique caractérisée par une forte minorisation et une forte ségrégation, qu'on peut synthétiser en ayant recours à la notion de situation de banlieue. Elle s'observe notamment dans les pratiques matrimoniales de groupes issus de l'immigration dans un contexte post-migratoire, comme le soulignent les lignes suivantes :

Le présent article postule la transformation progressive de l'endogamie dans les groupes minoritaires des sociétés post-migratoires. Cette dernière est moins une stratégie matrimoniale consistant à organiser l'union matrimoniale au sein du groupe lignager (Levi-Strauss, 1971), qu'une référence normative régissant l'univers des valeurs familiales. Ainsi, ce concept de tradition anthropologique ne semble plus adéquat pour saisir les évolutions en cours dans le contexte post-migratoire. Aujourd'hui, des facteurs relevant des traditions matrimoniales des pays d'origine se combinent avec des facteurs sociaux spécifiques aux conditions matérielles et résidentielles des populations immigrées installées dans les sociétés occidentales. Les mécanismes observés en France constituent un exemple pertinent des processus qui s'observent de manière plus générale dans toute société ayant connu dans la deuxième moitié $d u X X^{e}$ siècle des migrations de main d'œuvre ou d'anciens pays colonisés. L'ancienneté de l'installation, la valorisation du métissage et les relations entretenues depuis l'école tendent à favoriser la formation des unions mixtes. Simultanément, le maintien dans un statut de minorité et la stigmatisation qui l'accompagne, la relégation résidentielle dans des espaces urbains ségrégués, désignés "banlieues » en France ainsi que les inégalités et injustices subies par les populations "visibles» et/ou musulmanes contribuent à la formation d'un marché matrimonial ethnique. (Santelli, Collet, $2011: 330$ )

Force est de constater que les points de vue sur la question de l'endogamie reflètent l'absence de consensus social sur la question. La prise en compte des travaux de sociolinguistique et de didactique sur la diversité linguistique et culturelle, les situations plurilingues, les langues familiales des migrants, le / les statut(s) du français et des autres langues présentes dans les zones urbaines sensibles pourrait être une façon de faire contrepoids aux risques de repli communautaire et d'instrumentalisation à des fins politiques des questions linguistiques

\footnotetext{
De la même façon, les sociologues montrent aujourd'hui la persistance et la reconfiguration des liens de solidarité de type communautaire. Au sein des familles, les femmes fournissent des aides indispensables aux personnes dépendantes (les jeunes, les malades et les personnes âgées en particulier). La solidarité et le lien social ne sont pas du seul ressort de l'État. Au contraire, la pluralité des formes de solidarité correspond à la multiplicité des liens sociaux. Ainsi, l'objectif de renforcer le lien social passe par une action visant à accentuer chacun des liens sociaux en organisant leur mise en relation » (Ladouceur, 2008).

${ }^{21}$ Le Monde du 6 juillet 2004.
} 
(Bertucci, Corblin, 2007). On fera l'hypothèse que le repli communautaire peut résulter de l'identification des populations non autochtones aux espaces des banlieues défavorisées qui deviennent de ce fait des espaces de relégation auxquels sont conférés de surcroit une dimension identitaire.

\section{II.3.2 L'identité territorialisée}

Si on adhère à l'idée que la position spatiale d'un individu a une influence sur son comportement sociétal et que la somme des comportements individuels a aussi des conséquences collectives (Bussi, 2006: 66), la position spatiale d'un individu peut être comprise comme l'ensemble des relations conscientes et inconscientes entre lui et l'espace dans lequel il évolue. On postulera qu'existent dans ces cités des solidarités communautaires puissantes, qui sont au fondement d'un processus de patrimonialisation original qu'on va tenter de montrer en partant des processus d'identification juvéniles à un territoire.

Dans la cité, les jeunes s'identifient à un espace, à un territoire. Ce processus a été analysé comme une forme d'expérience identitaire élémentaire (Dubet, Lapeyronie, 1992 : 185). Cette appartenance souvent forcée, vécue comme stigmatisante est parfois le seul type d'identification possible dont les jeunes disposent ${ }^{22}$.

Ce processus d'identification/appartenance peut être analysé comme une forme de retournement du stigmate ${ }^{23}$ (Goffman, 1975) ou comme une façon d'imposer des liens égalitaires. Dans tous les cas, il s'agirait d'une identité territorialisée. Caractérisée également par un fort ancrage communautaire, elle se structure par une forme de lien social, qui a été décrite par Oscar Lewis à travers la notion controversée de culture de la pauvreté. On soulignera que cette notion fait l'objet d'un vif débat dans la communauté scientifique ${ }^{24}$ qui ne lui ménage pas ses critiques. Elle est cependant convoquée de manière récurrente dans les travaux des spécialistes. Le terme pauvreté, peut, en effet, être discuté, du point de vue théorique, car il peut paraitre trop connoté. Les termes exclusions et cultures au pluriel pourraient lui être substitués.

\section{La culture de la pauvreté}

\section{III.1 Une notion mise en débat}

Cette thématique de la culture de la pauvreté a été analysée dans les années soixante par Lewis dans un ouvrage intitulé: Les Enfants de Sanchez. Autobiographie d'une famille mexicaine. Pour Lewis, si la pauvreté apparait en premier lieu comme un état objectif mesurable en termes de revenus, elle peut se comprendre aussi comme un état culturel. Le déficit économique ne suffit pas à la décrire. Les groupes concernés s'organisent en communautés

\footnotetext{
${ }^{22}$ Dubet et Lapeyronie, Ibid.

${ }^{23}$ On rappellera la définition du stigmate : «C'est Erving Goffman qui a fait du stigmate (étymologiquement une marque durable sur la peau) un concept sociologique, en l'étendant à tout attribut social dévalorisant, qu'il soit corporel ou non - être handicapé, homosexuel, juif, etc. Le stigmate n'est pas un attribut en soi : il se définit dans le regard d'autrui. Il renvoie à l'écart à la norme : toute personne qui ne correspond pas à ce qu'on attend d'une personne considérée comme « normale » est susceptible d'être stigmatisée. Le stigmate s'analyse donc en termes relationnels. Il renvoie autant à la catégorie à proprement parler qu'aux réactions sociales qu'elle suscite et aux efforts du stigmatisé pour y échapper. Goffman distingue donc tout un jeu possible de négociations identitaires « lorsque la différence n'est ni immédiatement apparente, ni déjà connue, lorsqu'en deux mots, l'individu n'est pas discrédité, mais bien discréditable ». La personne stigmatisable s'attache au contrôle de l'information à l'égard de son stigmate (le cacher, le dire à certains, le révéler); la personne stigmatisée doit gérer la tension entre la norme sociale et la réalité personnelle (se voir confrontée aux réactions gênées de son entourage). Elle se trouve généralement réduite à son stigmate : toutes ses actions sont interprétées à travers ce prisme. Dès lors, elle est séparée des normaux. » (Rostaing, in Paugam, 2015 : 100)

${ }^{24}$ Le choix est fait ici de se référer à cette notion, qu'on estime ne pas pouvoir éluder, compte tenu de la place qu'elle occupe dans le champ des études consacrées à ces questions.
} 
structurées, dotées d'une appréhension propre de la vie. Cette culture possède des caractères propres, très stables et dotés d'une forme d'universalité, lesquels «transcendent les distinctions » (Lewis, $1972: 30$ ). On compte au nombre de ces caractéristiques la sociabilité communautaire et l'attachement au groupe de pairs chez les plus jeunes.

Cette conception ne fait pas l'unanimité. Lapeyronnie notamment considère qu'il n'y a pas de mode de vie marginal, relevant d'un ensemble de normes ${ }^{25}$ culturelles construites « comme un mode d'adaptation à l'exclusion sociale » (1993:253) et qu'une certaine unité des conduites ne signifie pas forcément une unité culturelle de la pauvreté, de la marginalité (Ibid.). La culture de la pauvreté a été critiquée par certains chercheurs anglo-saxons. Valentine souligne qu'elle revient à «blâmer la victime » ou à considérer les pauvres comme étant « responsables » des faits (1968) ; pour Goode et Eames la notion s'inscrit dans le cadre des mythes qui justifient les inégalités sociales. En 2010, en France, Lagrange a repris cette notion pour analyser les constructions culturelles des quartiers sensibles et insiste sur les processus d'ethnicisation qui y sont à l'œuvre, en y lisant une tension entre les héritages culturels des migrants, la tentation, qui les habite, d'un retour à la tradition et les contradictions de la société d'accueil. Sans développer davantage du fait des limites imparties par le cadre de cet article, on observera que, sous son côté opératoire, qui donne l'impression d'un cadre permettant une saisie d'ensemble du problème, la notion est d'un maniement problématique. Les spécialistes français de la pauvreté (Paugam, 2000 ; 2005 ; Lapeyronie, 2008) voient essentiellement le problème en termes socioéconomiques et non en termes de culture.

\section{III.2 Une approche de la pauvreté controversée}

On retiendra néanmoins le fait qu'ils soulignent la diversité des «configurations sociales » ${ }^{26}$ qui caractérisent la pauvreté (Paugam, 2012) aussi bien en France qu'en Europe comme en témoignent les propos suivants de Serge Paugam :

\footnotetext{
${ }^{25}$ On proposera la définition suivante : «Une des principales réflexions de la sociologie, depuis sa création, consiste à comprendre comment sont intériorisées les normes sociales. Selon Émile Durkheim, l'individu est certes susceptible de vouloir se singulariser par rapport à ses semblables mais sa conscience reste marquée par les normes qui enserrent le bien et le mal de la société dans laquelle il se trouve. C'est au travers des différentes instances de socialisation qu'il traverse (la famille, l'école, le travail, etc.) que l'individu intègre progressivement ce que le groupe auquel il appartient considère comme "normal" ou "anormal", conforme ou non à ses valeurs. C'est le propre des sociétés modernes que de produire des consciences individuelles qui ne sont pas un reflet mécanique des consciences collectives. Des failles dans le processus de socialisation, ou bien le fait d'être socialisé dans des univers contradictoires peuvent conduire un individu à se reconnaitre dans des ensembles normatifs différents, voire antagoniques. L'étude des normes sociales peut alors se faire en creux : il ne s'agit plus de décrire à quel(s) ensemble(s) de normes s'attachent telle société donnée, et donc tel groupe dominant en son sein ; mais de se pencher sur ce qui, dans une société, est considéré comme déviant : toute pratique ou toute identité perçue comme anormale, du fait des rappels à l'ordre et des éventuelles sanctions qu'elle suscite, informe ainsi sur ce qui fonde le "normal". » (Clair, in Paugam, 2015 : 83-84)

${ }^{26}$ On proposera la définition suivante de la notion de configuration sociale : « Le terme configuration a plusieurs acceptions en sociologie. Le terme renvoie cependant, d'abord, à la théorie élaborée par le sociologue allemand Norbert Elias. Ce concept signifie que la société est un réseau d'interdépendances entre individus. La société n'est donc pas une substance pas plus que l'individu ne saurait être isolé des chaines d'interdépendances dans lesquelles il s'inscrit. Pour saisir la société, le raisonnement doit être relationnel, comme l'objet qu'il vise à appréhender. Parler de configuration permet de mettre en lumière que la dépendance réciproque entre les individus est « la matrice constitutive de la société ». Les actions individuelles dépendent les unes des autres comme dans un jeu d'échecs par exemple où le déplacement d'un pion va modifier les actions possibles pour tous les pions. Ce concept renvoie donc à une conception fondamentalement relationnelle de la société. D'où l'erreur que fait selon Elias le sens commun (mais aussi la philosophie classique et une partie de la sociologie) lorsqu'il considère l'individu comme une réalité séparée alors même que celui-ci est le produit d'un processus de civilisation des mœurs et d'un développement déterminé des chaines d'interdépendance. Les sociologues font une erreur symétrique lorsqu'ils accordent un privilège à la société en la concevant comme ayant une réalité propre. Pour Elias, la configuration individu-société et ses évolutions est ce que le sociologue doit décrire et analyser car ces relations sont aussi réelles que les parties qu'elles relient. Dans cette perspective, le tissu de relations dont est faite la société s'apparente à
} 
Dans une recherche comparative menée principalement en Europe, j'ai pu toutefois vérifier que la pauvreté disqualifiante n'était qu'une forme élémentaire de la pauvreté parmi d'autres et que le cadre analytique devait être enrichi pour analyser les variations de la pauvreté dans l'espace et le temps (Paugam, 2005). Si la disqualification sociale a une probabilité plus élevée de se développer dans les sociétés "post-industrielles", notamment dans celles qui sont confrontées à une forte augmentation du chômage et des statuts précaires sur le marché du travail, il faut en effet se référer à d'autres concepts pour analyser des configurations sociales différentes. A revenu égal, être pauvre dans le Mezzogiorno n'a pas le même sens qu'être pauvre dans la région parisienne. Être pauvre dans le nord de la France dans les années 1960 n'avait pas non plus la même signification qu'être pauvre aujourd'hui dans la même région.

Serge Paugam, observe plus bas dans le même texte qu'il parait possible de parler d'une forme sui generis et disqualifiante (Ibid.) de pauvreté, qui peut être définie comme une « configuration sociale durable » comme l'indique le texte ci-après.

Le groupe des pauvres peut évidemment être défini en tant que tel à partir d'une mesure objective qui peut paraitre unanimement acceptable et s'imposer à tous comme un étalon universel, mais que signifie cette mesure si l'on n'interroge pas en même temps les représentations sociales et les expériences vécues de la pauvreté? Il faut surtout retenir qu'une forme élémentaire de la pauvreté correspond à un type de relation d'interdépendance suffisamment stable pour se maintenir durablement et s'imposer comme une unité sui generis, distincte des éléments individuels qui le caractérisent. Elle traduit un état d'équilibre relativement cristallisé des relations entre des individus inégaux (des pauvres et des non-pauvres) à l'intérieur d'un système social formant un tout. Au regard des évolutions constatées depuis vingt ans et de cette recherche comparative, il apparait que la pauvreté disqualifiante est désormais en France, mais aussi dans d'autres pays européens, une configuration sociale durable, dont on ne sortira qu'au prix d'efforts collectifs pour repenser le lien social et envisager des réformes profondes visant à assurer l'intégration solidaire, non pas seulement des pauvres et des assistés, mais de tous les membres de la société. (Paugam, 2012)

Cette idée qu'il existe une forme irréductible de pauvreté caractérisée par sa stabilité illustre l'ambigüité du statut de cette notion dans le champ des travaux consacrés à la question, sans se dissimuler ses limites évoquées précédemment, comme l'indiquent les faits contemporains qu'on peut convoquer pour illustrer ce point. On se réfèrera aux pages du Monde daté des $1^{\mathrm{er}}$ et 2 novembre 2016 (pp. 8-9) consacrées à la pauvreté d'abord dans le dossier conçu par Isabelle Rey-Lefebvre, composé des articles intitulés Face à la pauvreté, " on sent un vent mauvais » (p. 8), L'accueil d'urgence est saturé en dépit des efforts de l'État (p. 9) et de Témoignages de personnes sans domicile fixe (p. 8), ensuite dans l'article de Frédéric Cazenave, Ceux que cache la forêt (p. 8) qui aborde la question des personnes sans domicile fixe installées de manière durable dans le Bois de Vincennes à la proximité de Paris, enfin dans la section Débats et

un jeu, c'est-à-dire à une compétition porteuse d'une évolution. Le jeu est une «donnée élémentaire ». La métaphore du jeu indique que la vie sociale est concurrentielle et que les différents partenaires sont dans des relations d'interdépendance qui se stabilisent parfois dans un « équilibre des forces ». L'évolution des sociétés est expliquée par les modifications de cet équilibre par les actions individuelles et les effets qu'elles engendrent comme des réactions en chaine. Ces réactions en chaine modifient à leur tour le jeu et les joueurs. En s'appuyant sur la métaphore du jeu, Norbert Elias définit dans le terme de configuration : «la figure globale toujours changeante que forment les joueurs ; elle inclut non seulement leur intellect, mais toute leur personne, les actions et les relations réciproques. [...] Cette configuration forme un ensemble de tensions ». Largement supplantée par le concept de champ élaboré par Bourdieu pour décrire un système de relations dans lequel la domination est première et s'impose aux dominés sans qu'ils s'en aperçoivent, la façon dont Elias pense le jeu concurrentiel connait un renouveau d'intérêt en France depuis les années 1990. Son approche est notamment mobilisée par des sociologues comme Bernard Lahire, plus soucieux de ne pas écraser la réflexivité des acteurs tout en maintenant la contrainte du monde social. » (Duvoux, in Paugam, 2015 : 52-54) 
analyses, le texte d'Éric Pliez, N'attendons pas le premier mort pour augmenter l'hébergement d'urgence (p. 12). Le film Versailles (2008) réalisé par Pierre Schoeller illustre à travers une fiction la situation des sans-abris du bois de Vincennes; le documentaire de Claus Drexel (2013), Au bord du monde, aborde cette question de manière plus générale au-delà de la zone du bois de Vincennes et en donnant la parole à ces derniers. On peut également penser au film plus ancien d'Agnès Varda, Sans toit ni loi (1985), qui décrit le processus de marginalisation progressive et de décrochage d'une jeune errante jusqu'à sa mort.

On conclura cette approche de la notion de culture de la pauvreté en nous demandant si l'ambiguïté de la définition ne vient pas des tensions inscrites dans la notion de culture ellemême.

\section{III.3 La culture : une notion ambigüe}

La notion renvoie à la fois " à l'ensemble des symboles, des significations, des valeurs et des manières de faire propres à un groupe et au domaine spécialisé des activités expressives, savantes et populaires » (Paugam, 2015 : 59). Elle est utilisée aussi bien dans le cadre de l'analyse des «grandes thématiques sociologiques ${ }^{27}$ que pour celle des productions de l'activité ou de l'industrie culturelles ${ }^{28}$ (Ibid.). Dans la perspective de Bourdieu, la notion de culture illustre les rapports de domination et d'aliénation et les résistances à celles-ci, observables dans le champ de la culture (Paugam, 2015 : 60). On évoquera à cet égard les notions de «violence symbolique et de légitimité culturelle » développées par Bourdieu dans l'ouvrage intitulé La Distinction. Critique sociale du jugement (1979). Le courant des cultural studies (CS) a mis en évidence dans une perspective différente les modalités propres des «sociabilités» et des «cultures populaires » ${ }^{29}$ (Mattelart, Neveu, 2003: 31). Certains théoriciens de ce courant, notamment Williams (1958) et Thompson (1963) ont beaucoup insisté sur les antagonismes qui existent entre l'économie et la culture (op. cit. : 23) en se situant dans une perspective marxiste. Cette polysémie conduit Serge Paugam à écrire que la notion de culture est utilisée à la fois comme "variable explicative et comme variable expliquée » de l'analyse sociologique (Ibid.). Ceci amène à dire que la notion possède un statut d'indétermination qui la rend instable et hétérogène, c'est la raison pour laquelle Paugam préfère parler «d'état » ou de « configuration sociale » $(\text { Paugam, 2012) })^{30}$ et ne se réfère pas à la culture du groupe qu'il évoque. La stabilité évoquée dans le texte de Paugam est due à la forte précarisation résultant des importantes difficultés socioéconomiques du groupe concerné et non à une essentialisation des "valeurs » ou "manières de faire » de ce groupe pour reprendre les termes mêmes de Paugam cités au début du paragraphe. Cette précarisation conduit à une installation durable dans la précarité des acteurs évoqués et ce en raison de nombreux indicateurs négatifs qui compromettent une insertion sociale réussie, notamment l'accès à l'éducation, à l'emploi, au logement et les conditions de vie ${ }^{31}$ d'une manière générale (France Stratégie, $2015:$ 1). On soulignera également que les explications données sont les résultantes de la déprolétarisation ${ }^{32}$, des restructurations du travail et de l'emploi ${ }^{33}$, du poids du

\footnotetext{
${ }^{27}$ « Stratifications, inégalités, institutions, mouvements sociaux » (Paugam, $\left.2015: 59\right)$. Voir aussi sur les questions de « violence symbolique et de légitimité culturelle», Bourdieu (1979).

${ }^{28}$ «Les arts, les médias de masse, la science, l'industrie des loisirs et du divertissement, la religion » (Paugam, $2015: 59)$.

${ }^{29}$ Et au-delà des cultures jeunes.

${ }^{30}$ Comme on le lit dans la citation supra.

31 Voir supra, §I.1.

${ }^{32}$ Définie comme la sortie durable du marché du travail salarié d'une fraction importante de la classe ouvrière, qui a de fortes difficultés à retrouver un emploi stable (Rifkin, 1996 ; Wacquant, 2006 : 274).

${ }^{33}$ Le phénomène est apparu dans les années soixante-dix et s'est traduit par la dilution de la classe ouvrière et l'affaiblissement des liens sociaux fondés sur l'identification à une communauté de destins.
} 
chômage et de l'apparition d'une forte proportion de travailleurs pauvres depuis la fin des trente glorieuses ${ }^{34}$. Ces derniers constituent un salariat désocialisé, notamment à cause de l'affaiblissement des mécanismes de protection sociale et de leur désorganisation, liée à la disparition du cadre social et temporel commun fourni par l'emploi (Wacquant, 2006 : 274275). On postulera donc que si les habitants des quartiers précarisés ont en commun la faiblesse de leurs revenus, ils n'en constituent pas pour autant un peuple ouvrier et solidaire (La Mache, $2005: 23$ ) et le lien qui les unit n'est ni un lien politique de type contractuel, qui poserait une volonté commune ou générale (Mengue, 2008 : 98) ni un lien issu d'un vouloir-vivre ensemble (Bertucci, 2012: 19-20). C'est la raison pour laquelle il semble nécessaire d'adopter une certaine distance vis-à-vis de la notion de culture de la pauvreté et de poser la question de l'existence du risque d'une fragmentation du lien social liée à l'existence possible de formes de désaffiliation dans les zones urbaines sensibles. Il semble en effet que le discours commun ait tendance à considérer les zones urbaines sensibles comme des espaces où le sens de la vie collective s'est dilué, le lien social s'est fragmenté et dans lesquels la violence peut se développer, dans la mesure où ce sont les flambées de violence qui conduisent à la médiatisation de ces espaces et en font un problème social.

\section{III.4 Les phénomènes de fragmentation du lien social. Le risque de désaffiliation}

La désaffiliation ${ }^{35}$ a été décrite (Castel, 1995) comme la résultante des difficultés économiques et du chômage. Elle permet de définir la marginalisation qui en résulte et en particulier le chômage et la précarité des travailleurs pauvres. Pour Castel, la désaffiliation est une "notion alternative» à celle d'exclusion (Martin, 2015 : 61). L'exclusion comme la désaffiliation sont des «marqueur[s] » de la "dissolution du lien social » (Guibet Lafaye, 2012 : 16). La désaffiliation serait la conséquence d'un double « déficit» (Martin, $2015: 62$ ), à savoir un « déficit de filiation » et un « déficit d'affiliation » (Ibid.). Le premier renvoie « au déficit d'inscription dans des liens sociaux primaires (notamment familiaux)» (Ibid.). Le second concerne le manque "d'inscription dans des formes collectives de protection et, en particulier, la protection issue des collectifs de travail » (Ibid.).

L'analyse de ce processus a pour objectif de mettre en évidence les mécanismes :

qui font transiter les individus de l'intégration à la vulnérabilité, ou basculer de la vulnérabilité dans l'inexistence sociale [...], la désaffiliation consistant alors en un éloignement progressif des réseaux principaux d'activité. [...] ; une dialectique de la socialisation / désocialisation, de l'intégration / non intégration. (Guibet Lafaye, 2012 : 40-41)

Cette notion doit être envisagée avec attention car elle renvoie à un courant d'analyse de la sociologie française, qui s'intéresse de longue date aux " phénomènes dits de fragmentation » (Guibet Lafaye, 2012 : 34) du lien social, sachant que l'étude du lien social est un champ

\footnotetext{
${ }^{34}$ Période située de la fin de la seconde guerre mondiale au premier choc pétrolier, soit 1945-1974.

${ }^{35}$ On proposera la définition suivante : «Adoptant une posture critique à l'égard de la notion d'exclusion, qui connait au début des années 1990 une popularité considérable, Robert Castel propose dans plusieurs de ses textes une notion alternative, la désaffiliation, permettant d'éviter un certain nombre d'écueils. Pour Castel, la notion d'exclusion est un piège, du fait non seulement qu'elle fonctionne comme un mot-valise qui permet à la fois des usages divers (politiques, médiatiques et académiques), mais aussi de nommer une diversité de situations en gommant leurs spécificités. Pour Castel, il importe de dépasser le constat des «états de dépossession » pour analyser les mécanismes qui génèrent ce phénomène. [...] Il propose de distinguer deux axes permettant de penser les situations de dénuement : un axe d'intégration - non-intégration par le travail et un axe d'insertion - noninsertion dans une sociabilité socio-familiale. En somme, pour définir la désaffiliation, il est tentant de recourir à deux figures : déficit de filiation et déficit d'affiliation. » (Martin, in Paugam, 2015 : 60-61)
} 
d'étude particulièrement fécond pour cette discipline (op. cit. : 19) ${ }^{36}$. Elle fait écho à l'image de la fracture sociale, à laquelle le discours politique a très souvent recours, elle est également présente dans le discours commun. Des théoriciens comme Alain Touraine ou Michel Wieviorka la convoquent dans leur analyse des phénomènes d'exclusion et la perçoivent comme une rupture dans le continuum du tissu social contemporain (Guibet Lafaye, 2012 : 32).

\section{Conclusion}

Pour conclure, on soulignera que cette précarité, ajoutée à la segmentation de l'espace contribue à assigner à résidence des familles qui n'ont aucun autre choix résidentiel (Sintomer, Bacqué, 2002 : 102). De là, peut naitre un esprit de scission, notamment chez certains jeunes, sans qu'on puisse généraliser l'idée qu'il existe une contre-affiliation globale en l'état actuel des choses (Sintomer, Bacqué, 2002 : 107), laquelle aurait pour conséquence l'adhésion à d'autres systèmes de valeurs (Bertucci, 2009). Par ailleurs, on peut se demander si la conception française de l'intégration en maintenant l'assujettissement aux modèles institutionnels n'est pas contre-productive et ne contribue pas au maintien des phénomènes de stigmatisation / minorisation. Décrite comme nationaliste-républicaine (Lorcerie, 1994), cette position a créé un consensus général, mais a empêché toute expression de la différence, perçue immédiatement comme dissidente et portant les germes du communautarisme (Baudin, Genestier, op . cit. : 115). On peut donc se demander si, au fond, cet idéal d'intégration au nom de l'assimilation, n'a pas frappé de manière normative toutes les manifestations de différenciation, en privant les individus concernés de toute marge de manœuvre et d'autonomie (Rudder, 2002 : 116) et en stigmatisant toute expression de l'altérité.

\section{Bibliographie}

Baudin G., Genestier P., 2002, Banlieues à problèmes : la construction d'un problème social et d'un thème d'action publique, Paris : La Documentation française.

Bertucci M.-M., 1995, Contribution à une étude des dysfonctionnements linguistiques chez les élèves créoles de la Réunion, Villeneuve d'Ascq : ANRT.

Bertucci M.-M., 2006, Plurilinguisme et altérité. Français. École. Politiques linguistiques éducatives. Document de synthèse présenté pour l'Habilitation à diriger des recherches. Université de Tours.

Bertucci M.-M., Corblin C. (Coord.), 2007, Enseigner les langues d'origine, Le Français aujourd'hui, $\mathrm{n}^{\circ} 158$, Paris : Armand Colin.

Bertucci M.-M., 2009, «L'intégration des migrants dans la société française : situation linguistique, territorialisation, minorisation? », in L. Toft et L. Verstraete-Hansen (éds.), Une francophonie plurielle. Langues, idées et cultures en mouvement, Études Romanes, $\mathrm{n}^{\circ} 59$, Université de Copenhague : Museum Tusculanum Press, pp. 33-46.

Bertucci M.-M, 2012, «La notion de quartier populaire à l'épreuve de la modernité », in A. Lounici, N. Bestandji (dirs.), Dynamiques sociolangagières de l'espace algérois, Paris : L'Harmattan, pp. 17-35.

Bertucci M.-M., 2020, «Mémoires de l'immigration. Propositions pour une étude sociolinguistique des parlers des jeunes des cités urbaines sensibles de la France contemporaine », in Machado I. L., Proença Lara G. M., Turpin B. (dir.), Revista de

\footnotetext{
${ }^{36} \mathrm{C}$. Guibet Lafaye précise que la mise en relation des phénomènes d'exclusion / désaffiliation avec celle des liens sociaux parait être un indicateur pour mesurer le degré de « dissolution de la cohésion sociale » (2012: 19).
} 
estudos da linguagem, $\mathrm{n}^{\circ} 28-1$, université fédérale du Minas Gerais, faculté des Lettres, pp. 535-564, http://www.periodicos.letras.ufmg.br/index.php/relin/article/view/15496. Bourdieu P., 1979, La distinction. Critique sociale du jugement, Paris : éditions de Minuit.

Bussi M., 2006, "Géocratie ", in Séchet R. \& Veschambre V. (dirs.) Penser et faire la géographie sociale. Contributions à une épistémologie de la géographie sociale, Rennes : PUR.

Castel R., 1995, Les métamorphoses de la question sociale, Paris : Fayard.

Castra M., 2015, «Identité », in Paugam S., Les 100 mots de la sociologie, Paris : PUF, pp. 7273.

Castra M., 2015, « Socialisation », in Paugam S., Les 100 mots de la sociologie, Paris : PUF, pp. 97-98.

Clair I., 2015, « Normes », in Paugam S., Les 100 mots de la sociologie, Paris : PUF, pp. 8384.

Clair I., 2015, « Race et ethnicité », in Paugam S., Les 100 mots de la sociologie, Paris : PUF, pp. 112-113.

Cognet M., 2010, «Genre et ethnicité dans la division du travail en santé : la responsabilité politique des États », in L’homme et la société, n¹76-177, pp. 101-129.

Dorlin E., 2008, Sexe, genre et sexualités. Introduction à la théorie féministe, Paris : PUF.

Dubet F., Lapeyronnie D., 1992, Les quartiers d'exil, Paris : Fayard.

Duvoux N., 2015, «Configuration », in Paugam S., Les 100 mots de la sociologie, Paris : PUF, pp. 52-54.

Ferréol G., Jucquois G. (dirs.), 2003, Dictionnaire de l'altérité et des relations interculturelles, Paris : Armand Colin.

Frickey A. (dir.), 2005, Jeunes diplômés issus de l'immigration : insertion professionnelle ou discriminations, Paris : La Documentation française.

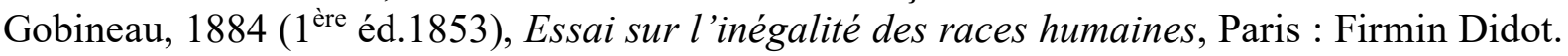
2 vol.

Goffman E., 1975, Stigmate les usages sociaux des handicaps, Paris : Éd. de Minuit,.

Goode J., Eames E., 1996, "An Anthropological Critique of the Culture of Poverty », in Gmelch G., Zenner W., Urban Life, Long Grove : Waveland Press.

Guillaumin C., 2002 [1992], L'idéologie raciste, Paris : Gallimard.

Hoggart, 1957, (Trad. fr. 1970), La Culture du pauvre, Paris : éditions de Minuit.

Joffe H., 2005, "L'Autre" et la construction identitaire : entre dynamiques psychiques et dynamiques sociales », in Sanchez-Mazas M., Licata L. (dirs.), L'autre. Regards psychosociaux, Grenoble : Presses universitaires de Grenoble. pp. 95-116.

Lagrange H., 2010, Le Déni des cultures, Paris : Seuil.

La Mache D., 2005, L'Art d'habiter un grand ensemble HLM, Paris : L'Harmattan.

Lapeyronnie D., 1993, La France et la Grande Bretagne face à leurs immigrés, Paris : PUF.

Lapeyronnie D., 2008, Ghetto urbain : ségrégation, violence, pauvreté en France aujourd'hui, Paris : Robert Laffont.

Lepoutre D., 1997, Cour de banlieue, codes, rites et langages, Paris : Odile Jacob.

Lewis O., 1972 [1963], Les enfants de Sanchez. Autobiographie d'une famille mexicaine, Paris : Gallimard.

Lorcerie F., 1994, «Les sciences sociales au service de l'identité nationale : le débat sur l'intégration au début des années 1990 », in Martin C. (dir.), Cartes d'identité. Comment dit-on nous en politique, Paris : Presses de la FNSP, pp. 245-281.

Lorcerie F., 2009, «Fractures sociales, fractures territoriales. L'École, son territoire et l'ethnicité », in Projet 312, pp. 64-71.

Martin C., 2015, «Désaffiliation », in Paugam S., Les 100 mots de la sociologie, Paris : PUF, pp. 61-62. 
Mattelart A., Neveu É, 2003, Introduction aux Cultural Studies, Paris : La Découverte.

Mengue P., 2008, Peuples et identités, Paris : éd. de La Différence.

Merton R. K., 1997 ( $3^{\mathrm{e}}$ éd.), Éléments de théorie et de méthode sociologique, Paris : Armand Colin.

Mucchielli L., Le Goaziou V., 2006, Quand les banlieues brûlent : retour sur les émeutes de novembre 2005, Paris : La Découverte.

Paugam S., 2000, La disqualification sociale : essai sur la nouvelle pauvreté, Paris : PUF.

Paugam S., 2005, Les formes élémentaires de la pauvreté, Paris : PUF.

Paugam S., 2015 [2010], Les 100 mots de la sociologie, Paris : PUF.

Rey H., 1996, La peur des banlieues, Paris : Presses de Sciences Po.

Rifkin J., 1996, La fin du travail, Paris : La Découverte.

Rostaing C. 2015, «Stigmate », in Paugam S., Les 100 mots de la sociologie, Paris : PUF, p. 100.

Sintomer Y., Bacqué M.-H., 2002, « Les banlieues populaires entre intégration, affiliation et scission », in Baudin G., Genestier P., Banlieues à problèmes : la construction d'un problème social et d'un thème d'action publique, Paris : La Documentation française, pp. 93-111.

Rui S., 2015, « Statut », in Paugam S., Les 100 mots de la sociologie, Paris : PUF, pp. 115-116. Rudder V. de, 2002, «Banlieues et immigration. Le social, l'urbain et la politique. Entretien avec V. de Rudder », in Baudin, G., Genestier, P., Banlieues à problèmes : la construction d'un problème social et d'un thème d'action publique, Paris: La Documentation française, pp.113-120.

Thompson E.P., 1975 [1963], The making of the english working class, Londres : Penguin book. Valentine V., 1968, Culture and Poverty, Critique and Counter-Proposals, Chicago: University of Chicago Press.

Van de Velde C., 2015, «Solidarité », in Paugam S., Les 100 mots de la sociologie, Paris : PUF, p. 99.

Wacquant L., 2006, Parias urbains. Ghettos. Banlieues. État, Paris : La Découverte.

Williams R., 1958 (Trad. fr. 1966), Culture and Society, 1780-1950, New York : Harper and Row.

Young R., 1995, Colonial desire : hybridity in theory, culture and race, London, New-York : Routledge.

\section{Sitographie $^{37}$}

Bertucci M.-M., (avec Assier J. et Chemblette E. coll.), 2009 et 2017, Mémoire de l'immigration. Vers un processus de patrimonialisation. Rapport de recherche incluant un corpus à l'intention du Ministère de la Culture et de la Communication et la Cité Nationale de l'Histoire de l'Immigration, 1 vol. (170 p.), disponible à la BNF depuis 2009 et sur le site du ministère de la Culture, mission à l'ethnologie depuis 2017, URL : https://www.culture.gouv.fr/Media/Thematiques/Patrimoineethnologique/Files/Rapports-de-recherche/2009/Ethno Bertucci 2009 517.pdf

Boucher M., 2012, « L'ethnicisation de la médiation sociale dans des "quartiers ghettos". Non, la politique des « grands frères » n'est pas morte!», Migrations Société n¹40, pp. 2534, URL : https://www.cairn.info/revue-migrations-societe-2012-2-page-25.htm

Cusset P.-Y., Garner H., Harfi M., Lainé F., Marguerit D., 2015, "Jeunes issus de l'immigration : quels obstacles à leur insertion économique ? ", La Note d'analyse hors

\footnotetext{
${ }^{37}$ Toutes les références de la sitographie sont actives au 23/02/2020.
} 
série. France Stratégie, Commissariat général à la stratégie et à la prospective, pp. 115, URL : http://www.strategie.gouv.fr

Tavan C., 2005, "Les immigrés en France : une situation qui évolue », INSEE Première, $\mathrm{n}^{\circ} 1042$, Paris : INSEE, URL : $\underline{\text { http://www.insee.fr/fr/ffc/docs ffc/IP1042.pdf }}$

Guibet Lafaye C., 2012, « Anomie, exclusion, désaffiliation : dissolution de la cohésion sociale ou du lien social ? », Pensée Plurielle, n²9, pp. 11-35. https://www.cairn.info/revuepensee-plurielle-2012-1-page-11.htm

Ladouceur B., 2008, " Serge Paugam, Le lien social », Lectures [En ligne], Les comptes rendus, mis en ligne le 28 aout 2008, URL : http://journals.openedition.org/lectures/653

Paugam S., 2012, "Les formes contemporaines de la disqualification sociale », http://ceriscope.sciences-po.fr/pauvrete/content/part5/les-formes-contemporaines-dela-disqualification-sociale

Rapport 2011 de l'ONZUS - Observatoire national des zones urbaines sensibles, URL: http://www.ville.gouv.fr/?rapport-2011-de-1-onzus-novembre

Santelli E., Collet B., 2011, « De l'endogamie à l'homogamie socio-ethnique : réinterprétations normatives et réalités conjugales des descendants d'immigrés maghrébins, turcs et africains sahéliens », Sociologie et sociétés, $\mathrm{n}^{\circ} 2$, pp. 329-354, URL: http://id.erudit.org/iderudit/1008249ar,

\section{Articles de presse}

\section{Le Monde du 6 juillet 2004}

Smolar P., « Les RG s'alarment d'un « repli communautaire » dans les banlieues », rapport de juin 2004 des Renseignements Généraux au Premier ministre.

Bernard P., Entretien avec Didier Lapeyronnie, « Le repli a lieu sur le ghetto, un lieu vide de sens $»$.

\section{Le Monde des $1^{\mathrm{er}}$ et 2 novembre 2016}

Rey-Lefebvre I., «Face à la pauvreté, « on sent un vent mauvais » », pp. 8-9.

Rey-Lefebvre I., «L'accueil d'urgence est saturé en dépit des efforts de l'État », p. 8.

Témoignages des sans domicile fixe, p. 8.

Cazenave, F. « Ceux que cache la forêt », p. 8.

Pliez É «N'attendons pas le premier mort pour augmenter l'hébergement d'urgence », p. 20.

\section{L'Histoire $n^{\circ}$ 306, février 2006, Noirs et Blancs. Apartheid, ségrégation, discrimination.}

Febvre C., Entretien avec Didier Fassin «Y a-t-il vraiment des discriminations raciales en France? », pp. 53-57.

\section{Filmographie}

\section{Drames}

Schoeller P., 2008, Versailles, Les films Pelleas, France, $113 \mathrm{mn}$.

Varda A., 1999, Sans Toit ni Loi, Ciné-Tamaris, France, 105 mn.

\section{Documentaire}

Drexel C., 2013, Au bord du monde, Daisy Day Films, France, 98 mn. 


\section{GLOTTOPOL}

Revue de sociolinguistique en ligne

Comité de rédaction : Michaël Abecassis, Salih Akin, Sophie Babault, Claude Caitucoli, Véronique Castellotti, Régine Delamotte, Robert Fournier, Stéphanie Galligani, Emmanuelle Huver, Normand Labrie, Foued Laroussi, Benoit Leblanc, Fabienne Leconte, Gudrun Ledegen, Danièle Moore, Clara Mortamet, Alioune Ndao, Isabelle Pierozak, Gisèle Prignitz.

Rédactrice en chef : Clara Mortamet.

Comité scientifique : Claudine Bavoux, Michel Beniamino, Jacqueline Billiez, Philippe Blanchet, Pierre Bouchard, Ahmed Boukous, Pierre Dumont, Jean-Michel Eloy, Françoise Gadet, Monica Heller, Caroline Juilliard, Jean-Marie Klinkenberg, Jean Le Du, Marinette Matthey, Jacques Maurais, Marie-Louise Moreau, Robert Nicolaï, Didier de Robillard, Paul Siblot, Claude Truchot, Daniel Véronique.

\section{Comité de lecture pour ce numéro :}

Mickael Abecassis, Michelle Auzanneau, Salih Akin, Céline Amourette, Gabriel Bergounioux, Philippe Boula de Mareuil, Catherine Brissaud, Aude Bretegnier, Maria Candea, Véronique Castellotti, Régine Delamotte, Jean-François De Pietro, Catherine Delarue-Breton, Anne Dister, Alexandre Duchêne, Valentin Feussi, Françoise Gadet, Stéphanie Galligani, François Gaudin, Monica Heller, Emmanuelle Huver, Caroline Juilliard, Abou Bakri Kebe, Jean-Marie Klinkenberg, Christian Lagarde, Foued Laroussi, Fabienne Leconte, Evelyne Lloze, Nolwenn Lorenzi, Gudrun Ledegen, Nadja Maillard, Marinette Matthey, Bruno Maurer, Véronique Miguel Addisu, Saskia Mugnier, Claire Oger, Marielle Rispail, Richard Sabria, Véronique Traverso, Christel Troncy, Cécile Van den Avenne. 